Portland State University

PDXScholar

\title{
The Effects of Neuron Degeneration and Methods of Neurogenesis
}

Jesus I. Martinez

Portland State University

Follow this and additional works at: https://pdxscholar.library.pdx.edu/honorstheses

Part of the Neuroscience and Neurobiology Commons Let us know how access to this document benefits you.

Recommended Citation

Martinez, Jesus I., "The Effects of Neuron Degeneration and Methods of Neurogenesis" (2020). University Honors Theses. Paper 909.

https://doi.org/10.15760/honors.930

This Thesis is brought to you for free and open access. It has been accepted for inclusion in University Honors Theses by an authorized administrator of PDXScholar. Please contact us if we can make this document more accessible: pdxscholar@pdx.edu. 
The Effects of Neuron Degeneration and Methods of Neurogenesis

by
Jesus Martinez
An undergraduate honors thesis submitted in partial fulfillment of the
requirements for the degree of
Bachelor of Science
in
University Honors
and
Psychology

Thesis Adviser

Bill Griesar

Portland State University 


\begin{abstract}
In the 1960's, Joseph Altman discovered the creation of new neurons continues well into the adulthood of mammals. His work lay dormant for several decades possibly as a result of limitations in technology or the limited perceived usefulness at the time. In the 1990's Altman's discovery gained newfound interest and has stayed in researcher's interest ever since. A combination of improved imaging techniques, a continued shift in the public image of mental disorders, and a desire to aid those suffering from neurodegenerative disorders has led to many attempts to parse out the details of neurogenesis. After a number of studies that explore only the quantity and density of neurogenesis, there has been a small surge in research on the usefulness of neurogenesis. It is well established that the human brain is more than capable of managing itself in terms of the space and use of all the cognitive real estate. Why, then, is it useful to aid the growth of new neurons? All throughout a person's development the brain naturally prunes neurons and in many cases of physical damage the brain's neuroplasticity is able to recover a wide range of functions that would otherwise be considered lost. Because of this it is important to categorize types of neurodegeneration and what the effects of that damage is. I will begin this literature review by categorizing the types of neurodegeneration then follow up on the various methods that have been found to promote neurogenesis. Finally, I will go over the effects of neurogenesis that have been researched thus far. It is easy to assume that we should always try to promote neurogenesis as a part of healthy brain growth and maintenance but this should first be confirmed through scientific method. It is entirely possible that attempting to promote neurogenesis could be harmful, or not produce any measurable difference in health except in the most extreme cases. This is why more research needs to be done before coming to any broad
\end{abstract}


conclusions. It should be proven that neurogenesis actually produces measurable differences in health and/or cognition and what kind of conclusions can be drawn from the creation of new neurons well into adulthood.

\section{The Various Types of Neuron Degeneration}

The source of neuron degeneration is important because it grants some insight into the type of damage being done. Some possible problems include: a general loss of neuron density, a decrease in the myelin sheaths, or a loss of a specific type of neuron. Certain sources of neuron degeneration also target very specific locations in the brain and can have very specific effects on behavior and cognition; for these types of neuron damage you can imagine something like the case of Phineas Gage. Phineas Gage suffered a traumatic injury via railroad spike to the brain. He was able to survive but suffered immense damage to his left frontal lobe which resulted in large changes in his personality. Disease, cancer, or other less physical force can just as easily cause massive neuron damage in specific areas of the brain.

\section{Physical Brain Trauma}

Expanding on that thought, imagine that a large area of your frontal lobe experienced mass neuron degeneration. The sort of effects of this sort of trauma are well documented. When considering this type of brain cell damage compared to damage as a result of poor health or disease, it is relatively simple. The difficulty is in determining exactly which parts of the brain are lost. Once that is identified, you would be able to predict most of the effects that you could expect to see by considering the function of the area that experienced damage. Most of the time you could reasonably conclude that if the area is gone or irreparably damaged, whatever function that area of cortex serves will no longer be something that is possible for that individual. At least 
initially, this kind of analysis would be mostly correct; for example, you could expect that in the case of a physical trauma that damaged Broca's area you could expect the individual to display certain behaviors like broken speech. Although simplifying brain function in this way has its issues, one type of physical trauma can be thought of as "where once there were neurons, now there are not." Now not all physical injuries are as simple as that but it would be quite rare for a physical injury to only specifically damage a part of the neuron as opposed to just destroying the whole. As a result, a study that focuses on damage of this kind will be limited in the same way that a study looking into the function of an area of cortex will be. While there is value in exploring the ways that this type of damage occurs, for the purposes of this paper I will focus on how the brain's plasticity is typically more essential than neurogenesis.

Currently there is no way to restore large areas of the cortex that have been removed or destroyed. Even the methods for neurogenesis that have been documented don't come anywhere near being able to restore extensive loss. Damage that is on the scale of Phineas Gage is unfortunately permanent. Still, through various studies we've learned that when there is no chance of direct replacement, the brain will attempt to cover any loss in cognitive function by rewiring whatever lost functions it can to living and working parts of the brain. The success of this neural plasticity is widely variable and has been studied on a case by case basis. Here you can spot trends in what the extent of these types of repairs is but the accuracy of these claims is limited because of both ethical implications and limited sample size in humans. It would not be right to intentionally cause damage to brains just to see the extent of neural plasticity. Thus we must rely on the limited data available as a result of accidents or from animal experiments. 
Neuron Degeneration as a Result of Stress and Disease

Neuron degeneration can come in more complicated forms than simply physical damage. Dementia, Parkinsons, depression, and tinnitus are all examples of very specialized types of neuron degeneration that can happen as a result of pre-existing medical conditions and stress from all avenues of life. Neuron degeneration in this sense can be better defined as the targeted destruction of a localized type of neuron or parts of neurons. Using tinnitus as an example, in many cases only the inner hair cells in the cochlea are affected and often only a certain subset of these cells, namely those located in the high frequency regions of the basilar membrane. In a study published this year it was found that neuroplasticity was partially responsible for the disorder. Non-classical auditory structures in the brain can link with cochlear hair cells possibly as an attempt to make up for structural damage to the cells causing tinnitus symptoms (Kaplowski \& Thompson, 2020). Regrowing the hair cells is something that is currently not possible and most efforts are to minimize symptoms. Hearing loss is closely tied to this issue as it also revolves around damaged cochlear cells. Treatment of these cells is also currently only experimental when not using more conventional methods such as ear implants or hearing aids. Still the suppression of the connection that forms between non-classical auditory structures and the ear cells might be a start towards a solution for tinnitus.

Expanding on the idea that neurogenesis is a prevalent process even in adulthood, memory issues might be able to benefit from the same processes that are responsible for the linking of non-hearing structures and the cochlear hair cells and neurons. Whereas in the 
example of tinnitus, neuroplasticity posed an issue because the new links formed cause issues, when it comes to learning and memory there is a lot to gain by forming new connections through both neuroplasticity and neurogenesis. Memory loss and learning issues usually go hand in hand because retention of information is an important part of learning. How one could come to have memory problems or learning problems is extremely varied. Glia are often targets of diseases, including Alzheimer's disease (AD), Parkinson's disease (PD), and multiple sclerosis (MS) (Song, et.al, 2020). Although all of these diseases have unique aspects, AD is nearly synonymous with loss of memory. Learning issues can result from damage to the prefrontal cortex, as a result of disease or physical trauma, inhibiting cognition and critical thinking. In the Journal of Neuropsychopharmacology, a study affirmed that stressful experiences negatively regulate normal amounts of neurogenesis that occur in the dentate gyrus of the hippocampus. This negative correlation is linked to poorer memory among other negative effects that come with major depressive disorder (MDD)(Hanson, Owens, \& Nemeroff, 2011). This study served as an affirmation for many people who had reported feeling "foggy headed" and "forgetful" after years of suffering from MDD. The same study also found a positive correlation between antidepressants and neurogenesis. Since then other studies have also looked into the healing effects that antidepressants have in relation to neurogenesis and many seem to be coming to the conclusion that this is most notable in treatment with serotonergic antidepressants. Over the nearly 10 years since Hanson, Owens, \& Nemeroff's study on the dentate gyrus and antidepressants there have been numerous studies and most have branched away from antidepressants. There are studies looking into similar results being achieved by all sorts of drugs with "antidepressant-like" effects and a promotion of neurogenesis. It should be noted most of 
the studies making these types of claims are exclusively in animals. Ketamine is a particularly popular subject for review at the time of writing this.

Other Proposed Methods for Neurogenesis

The effects of neuron degeneration are as varied as they can be. Anything that a neuron has a part in can be affected by neuron degeneration. Because of this, the literature surrounding neurogenesis can be just as varied. The most popular and well understood methods of neurogenesis tend to fall in one of two different categories: neurogenesis through drugs, and neurogenesis through self care. I have already explained some of the proposed methods of neurogenesis that are currently under heavy research. These mostly involve drugs and stem from the original study that looked into neurogenesis in adult mammals back in the 1960's. What I have noticed is that these studies were performed exclusively in animals, particularly guinea pigs. Not only that but the 1960's were also notorious for the overprescription of antidepressants which could have served as the inspiration for Altman's study. Also adding to the problem is that it is difficult to separate the effects of the drugs from the overall improvement in health, both mental and physical. The overprescription helps bring some more validity to the situation because it means that it's possible that some people who didn't struggle with depression still benefited from the antidepressants.

In any case, currently there are several avenues of neurogenesis being explored and many seem to be proving to be quite promising. Two examples of this involve the exploration of one's environment and how it correlates with hippocampal neurogenesis. One of these studies is an animal study on mice that is actually an expansion on a previous study which found that identical 
mice will develop different levels of exploratory behavior patterns. That basically means that some mice would explore more than others and they quantified how much they explored. The more they explored the more hippocampal neurogenesis they were able to record. Their follow up study allowed them to hypothesize that greater area coverage in their explorations of their environment correlated with plasticity (Freund et al., 2015). This is just one example of researchers attempting to parse out the detail from the taxi cab study from 2011. 4 years previously it was discovered that taxi cab drivers in London showed significantly more hippocampal neurogenesis than the average Londoner. The reason they cited for this neurogenesis is they had learned the layout of the complex city streets over the course of 3 or 4 years. Not only did these experienced taxi cab drivers show an increase in gray matter in their posterior hippocampus, they also noticed that besides being better at recalling information about London they were slower in acquiring new spatial knowledge compared to the control group (Woollett \& Maguire, 2011). Although there hasn't been an opportunity to recreate this study on humans in the field, many studies have attempted to recreate or expand on the results in labs and typically on animals. Often studies on neurogenesis tend to focus on hippocampal volume and memory and learning and this could be traced back to the taxi cab study by Woolett \& Maguire.

Physical health and exercise have also been shown to have positive effects on the overall brain health. Exercise in particular has been studied as a way to promote neurogenesis. It is already known that exercise has numerous cognitive benefits and one study sought to connect neurogenesis to those benefits. Hypothesising that the boosts to memory and learning that come from exercise, researchers identified a specific molecule, which is produced during exercise, they believe is important to the entire process of neurogenesis. This research was limited by the 
information and test subjects available to do human research on however this only leaves the question of to what extent the molecule and therefore exercise has on human neurogenesis (Liu \& Nussloc, 2018). Really, what findings like this point to is non-pharmaceutical methods of neurogenesis can be used as a way to prevent or even just lessen the effects of neurodegeneration. This is especially true of neurodegeneration that happens as a result of aging or poor heath. A published article from 2017 took a more in depth look at the effects of exercise over a human lifetime. What they found was that it had a positive impact no matter the age leaving only the question of how much it helped (Saraulli et al., 2017). This is a common conclusion in research on exercise. One possible explanation is that mental health is an individual experience and while it is possible to identify things that are generally helpful to everyone, the success will vary by individual. What I have noticed in these studies is the research participants are often a random sample. This has both positive and negative effects on the research. Positively, it shows that no matter the individual's personal issues exercise has been shown to be beneficial to all. Negatively, it makes the research appear less substantial because there isn't ever a huge conclusion. As a comparison to pharmaceutical research, it would be like giving anti-migraine medication to a random sample of people instead of just people who suffer from migraines and then finding only a slightly positive trend.

Physical health is no doubt important mental health and brain health in general. This is something that is often overlooked in practice despite having a good amount of research backing it. For years it's been known that maintaining good physical health has profoundly positive effects on mental health and this is not an exception when it comes to memory, learning, and neurogenesis. Earlier this year a study was published showing that increasing neurogenesis 
restores some contextual memory and learning; these two things are known to decline in old age but now researchers are finding that this doesn't have to be definitive and in some cases can even be preventable (Berdugo-Vega et al., 2020). These findings are very close to common diseases like Alzheimers and although it is unlikely that we will be able to repair massive damage through diet, exercise and learning exercises, we know that these activities in addition to more pharmaceutical methods can only aid in lessening the damage that is caused neurodegeneration of all types.

One thing that should be noted as well is there was recently a post mortem study that found no new neurons are formed in adulthood. This study examined the dentate gyrus region of the hippocampus and although this claim is controversial among academics it does show that there is a gap in the literature. There are researchers who are dedicated to studying neurogenesis and if there are others trying to disprove neurogenesis in adulthood it shows that not everyone is on the same page yet. There are problems with the methods of the controversial study and their conclusions don't stand against all the research that shows neurogenesis is something that continues well into elderly years and possibly until death. I think the best response to research like this is to ask more questions both about their methods that led them to their opposing conclusion and also questions about how we are quantifying neurogenesis. Something I would like to see more of in the research is clear goals of why to pursue neurogenesis. There is literature that makes claims about improvements to memory and learning which are extremely valuable but better quantifying these results might lend more attention to findings about neurogenesis in adults. It would give people outside of academia another reason to care about 
their brain health and a reason to think about the findings of all the articles I discussed in this paper.

\section{Conclusions}

One thing that should be noted as well is there was recently a post mortem study that found no new neurons are formed in adulthood. This study examined the dentate gyrus region of the hippocampus and although this claim is controversial among academics it does show that there is a gap in the literature. There are researchers who are dedicated to studying neurogenesis and if there are others trying to disprove neurogenesis in adulthood it shows that not everyone is on the same page yet. There are problems with the methods of the controversial study and their conclusions don't stand against all the research that shows neurogenesis is something that continues well into elderly years and possibly until death. I think the best response to research like this is to ask more questions both about their methods that led them to their opposing conclusion and also questions about how we are quantifying neurogenesis. Something I would like to see more of in the research is clear goals of why to pursue neurogenesis. There is literature that makes claims about improvements to memory and learning which are extremely valuable but better quantifying these results might lend more attention to findings about neurogenesis in adults. It would give people outside of academia another reason to care about their brain health and a reason to think about the findings of all the articles I discussed in this paper. 
Throughout my exploration in the academic literature available as of writing I have found that the avenues for neurogenesis are vast and varied. There are ways to promote neurogenesis in adults that involve drugs, physical activity, mental exercise, and social inclusion. All of these have shown some degree of success which is an important conclusion to make because it means that there can be less compromise when it comes to treating individuals. The research presented above, and by extension any future research looking into how to promote neurogenesis, gives us the tools to tailor people's recovery in order to target not just emotional issues but neurochemical/cognitive issues. The work done by the researchers exploring non-pharmaceutical methods of neurogenesis in the articles above should not be used as a replacement for existing treatments for depression, dementia, and other conditions but rather as a supplement. The key takeaway should be as follows: neurodegeneration is an issue that comes in addition to common mental health issues and neurogenesis might be able to help to relieve the symptoms of many mental health issues and diseases. It is also important to remember, there are many methods to promote neurogenesis and not all involve pharmaceuticals and medical procedures.

What I would suggest is a change in focus from the drug aspect of neurogenesis and more so to focus on a healthy body and mind as a whole. The human brain has already proven that it is exemplary in promoting not only the generation of new neuron tissues but also in the rewiring of existing pathways in order to redevelop a healthier brain. Currently there are many more studies looking into drugs that interact positively with antidepressants in order to promote neurogenesis and reverse the effects of depression rather than fixing the root of the problem itself. 
Abbott, Louise C., and Fikru Nigussie. "Adult Neurogenesis in the Mammalian Dentate Gyrus.” Anatomia, Histologia, Embryologia 49, no. 1 (January 2020): 3-16. https://doi.org/10.1111/ahe.12496.

Altman, Joseph, and Gopal D. Das. "Postnatal Neurogenesis in the Guinea-Pig." Nature 214, no. 5093 (June 1967): 1098-1101. https://doi.org/10.1038/2141098a0.

Barberis, Andrea. "Postsynaptic Plasticity of GABAergic Synapses." Neuropharmacology 169 (June 2020): 107643. https://doi.org/10.1016/j.neuropharm.2019.05.020.

Berdugo-Vega, Gabriel, Gonzalo Arias-Gil, Adrian López-Fernández, Benedetta Artegiani, Joanna M. Wasielewska, Chi-Chieh Lee, Michael T. Lippert, Gerd Kempermann, Kentaroh Takagaki, and Federico Calegari. "Increasing Neurogenesis Refines Hippocampal Activity Rejuvenating Navigational Learning Strategies and Contextual Memory throughout Life." Nature Communications 11, no. 1 (December 2020): 135. https://doi.org/10.1038/s41467-019-14026-z.

Chohan, Muhammad O. "Deconstructing Neurogenesis, Transplantation and

Genome-Editing as Neural Repair Strategies in Brain Disease.” Frontiers in Cell and Developmental Biology 8 (March 13, 2020). https://doi.org/10.3389/fcell.2020.00116. Di Liegro, Schiera, Proia, and Di Liegro. "Physical Activity and Brain Health." Genes 10, no. 9 (September 17, 2019): 720. https://doi.org/10.3390/genes10090720.

Freund, J., A.M. Brandmaier, L. Lewejohann, I. Kirste, M. Kritzler, A. Krüger, N. Sachser, U. Lindenberger, and G. Kempermann. "Association between Exploratory Activity and Social Individuality in Genetically Identical Mice Living in the Same Enriched Environment." Neuroscience 309 (November 2015): 140-52.

https://doi.org/10.1016/j.neuroscience.2015.05.027.

Griesar, Bill. “Mental Health@ McMenamin's.” NW NOGGIN: Neuroscience Outreach Group (Growing in Networks), NW Noggin, 21 Apr. 2018, nwnoggin.org/2018/04/19/mental-health-mcmenamins/.

Guskjolen, Axel, Jonathan R. Epp, and Paul W. Frankland. "Hippocampal Neurogenesis and Forgetting." In The Hippocampus from Cells to Systems, edited by Deborah E. Hannula and Melissa C. Duff, 95-121. Cham: Springer International Publishing, 2017. https://doi.org/10.1007/978-3-319-50406-3 4. 
Hanson, Nicola D, Michael J Owens, and Charles B Nemeroff. "Depression, Antidepressants, and Neurogenesis: A Critical Reappraisal."

Neuropsychopharmacology 36 (September 21, 2011): 2589.

Kapolowicz, Michelle R., and Lucien T. Thompson. "Plasticity in Limbic Regions at Early Time Points in Experimental Models of Tinnitus." Frontiers in Systems Neuroscience 13 (January 24, 2020). https://doi.org/10.3389/fnsys.2019.00088.

Khoury, Rita, and George T. Grossberg. "Impact of Antidepressant Use on the Trajectory of Alzheimer's Disease: Evidence, Mechanisms, and Therapeutic Implications.” CNS Drugs 33, no. 1 (January 2019): 17-29. https://doi.org/10.1007/s40263-018-0590-9. Kozareva, Danka A., John F. Cryan, and Yvonne M. Nolan. "Born This Way: Hippocampal Neurogenesis across the Lifespan.” Aging Cell 18, no. 5 (October 2019).

https://doi.org/10.1111/acel.13007.

Liu, Patrick Z., and Robin Nusslock. "Exercise-Mediated Neurogenesis in the Hippocampus via BDNF." Frontiers in Neuroscience 12 (February 7, 2018): 52.

https://doi.org/10.3389/fnins.2018.00052.

Saraulli, Daniele, Marco Costanzi, Valentina Mastrorilli, and Stefano Farioli-Vecchioli.

“The Long Run: Neuroprotective Effects of Physical Exercise on Adult Neurogenesis

from Youth to Old Age." Current Neuropharmacology 15, no. 4 (April 11, 2017):

519-33. https://doi.org/10.2174/1570159X14666160412150223.

Snyder, Jason S., and Michael R. Drew. "Functional Neurogenesis over the Years."

Behavioural Brain Research 382 (March 2020): 112470.

https://doi.org/10.1016/j.bbr.2020.112470.

Song, Shanshan, Lanxin Luo, Baoshan Sun, and Dandan Sun. "Roles of Glial Ion

Transporters in Brain Diseases.” Glia 68, no. 3 (March 2020): 472-94.

https://doi.org/10.1002/glia.23699.

Xie, Wenjie, Xuping Li, Chao Li, Wen Zhu, Joseph Jankovic, and Weidong Le.

“Proteasome Inhibition Modeling Nigral Neuron Degeneration in Parkinson's Disease: Proteasome Inhibition Mouse Model of PD." Journal of Neurochemistry 115, no. 1

(October 2010): 188-99. https://doi.org/10.1111/j.1471-4159.2010.06914.x.

Yamada, Jun, and Shozo Jinno. "Potential Link between Antidepressant-like Effects of

Ketamine and Promotion of Adult Neurogenesis in the Ventral Hippocampus of Mice." Neuropharmacology 158 (November 2019): 107710.

https://doi.org/10.1016/j.neuropharm.2019.107710. 
Yau, Suk-yu, Ang Li, and Kwok-Fai So. "Involvement of Adult Hippocampal Neurogenesis in Learning and Forgetting." Neural Plasticity 2015 (2015): 1-13. https://doi.org/10.1155/2015/717958. 